\section{La emergencia}

\section{de la biologia}

en la escuela

colombiana:

una mirada

genealógica entre 1900-1930

\section{A emergência da biologia na escola colombiana: uma perspectiva genealógica entre 1900-1930}

\author{
The Emergence of Biology \\ in Colombian Schools-a \\ Genealogical Perspective, \\ 1900-1930
}

Este escrito presenta reflexiones sobre la biología en la escuela colombiana a partir del estudio de su historia, en particular su emergencia entre 1900 y 1930, periodo en el que es visible que la biología no siempre ha sido objeto de enseñanza escolar y que sus prácticas no se reducen a la repetición de las metodologías y conceptos de la biología como ciencia y de los biólogos como expertos. La ruta de relaciones analizada permite visibilizar que esta emergencia está relacionada con preocupaciones sobre la enfermedad, la supervivencia del niño, el desarrollo del cuerpo, la mejora de la raza, el régimen alimenticio y el incremento del amor por la naturaleza. Así, esta mirada no señala una línea continua de acontecimientos, sino su discontinuidad y muestra, por ejemplo, que la historia natural no desaparece con la emergencia de la biología, sus objetos de estudio en la escuela no son los mismos, además se problematiza la actualidad de su enseñanza a propósito de la sospecha sobre su desplazamiento por parte de las ciencias naturales.

\section{Palabras clave}

Biología en la escuela, emergencia, prácticas discursivas

\section{Resumo}

Este escrito apresenta reflexões sobre a biologia na escola colombiana a partir do estudo da sua história, em particular sua emergência entre 1900 e 1930, período em que é visível que a biologia não sempre há sido objeto de ensino escolar, e que suas práticas não se reduzem à repetição das metodologias e conceitos da biologia como ciência, e dos biólogos como expertos. A trilha de relações analisada permite visibilizar que a emergência esta relacionada com preocupações sob a doença, a supervivência da criança, o desenvolvimento do corpo, a melhora da raça, o regímen alimentício e o incremento do amor pela natureza. Assim, esta perspectiva não assinala uma linha contínua de acontecimentos, senão sua descontinuidade e mostra, por exemplo, que a história natural não desaparece com a emergência da biologia, seus objetos de estudo na escola não são os mesmos, além disso, se problematiza a atualidade de seu ensino ao propósito da suspeita sobre seu deslocamento pela parte das ciências naturais.

\section{Palavras chave}

Biologia na escola, emergência, práticas discursivas

\section{Abstract}

This paper is a reflection upon biology in Colombian schools, from the study of its history and, particularly, its emergence in 1900-1930. It can be seen that biology was not always a school subject matter. Its practice was restricted to repeating the methods and concepts of biologists - experts-, as well as those of biology as a science. From the analysis, you can state that the emergence of biology as a subject matter is related to concerns about illness, children's survival, the development of human body, the improvement of race, diet, and an increasing love for nature. From this perspective, the study states that events do not follow a continuum. For instance, it shows that natural history does not disappear when biology appears, since their objects of study at school are quite different. Furthermore, there is a reflection on its current teaching, taking into consideration that it might have been displaced by natural sciences.

\section{Key words}

Biology at school, emergence, discursive practices

Fecha de recepción: Marzo 2 de 2015

Fecha de aprobación: Mayo 22 de 2015

Pedagogía y Saberes No. 42 Universidad Pedagógica Nacional Facultad de Educación. 2015, pp. 145-151 
Síntesis del contenido curso de Biología primer año escuela normal:

El estudio de la vida y su desarrollo en el reino animal y vegetal.

La enseñanza biológica en este año debe estar principalmente orientada a

la defensa y a la vigorización racial por el conocimiento claro de los agentes

que se oponen y de los que favorecen esta vigorización, debe constituir,

por tanto, una preparación para el entendimiento de la higiene en el segundo año y también una introducción para la enseñanza agrícola.

Bernal, 1933

\section{Introducción}

$\mathrm{L}$ as reflexiones que se presentan a continuación se realizan con base en el trabajo de grado de maestría "Historia de los saberes escolares: la emergencia de la biología en la escuela colombiana 1900-1930", las discusiones de la línea de investigación "Trayectos y aconteceres: estudios del ser y el quehacer del maestro desde la pedagogía", los hallazgos del curso electivo "Historia de la biología en la escuela colombiana: una genealogía de la enseñanza de la biología y la biología escolar", y la práctica pedagógica de la autora.

Con esta propuesta investigativa, no se busca decir otras verdades acerca de la enseñanza de la biología, listar las leyes, o preinscripciones instauradas. Se busca problematizar la biología en la escuela, interrogar su necesidad, visibilizar las prácticas discursivas más allá de sus pretensiones de cientificidad, observar sus mutaciones, sus modulaciones, las reglas que van configurando el saber que de allí emerge, los enunciados que la hacen circular y los que ella hace circular, y la relación de estos con la constitución de sujetos y la formación de maestros.

De ese modo, las condiciones de emergencia no se constituyen solamente desde formas de saber como la ciencia o fuerzas de poder como las leyes, sino que tales condiciones también involucran todas las prácticas que son discursos y todos los discursos que son prácticas. De esta manera, la biología en la escuela no es una adaptación o réplica de la ciencia biológica pues se constituye desde prácticas y discursos que cambian, que son particulares y que no solo son producidos, sino que también producen, constituyéndose en fuerza. Así, se puede afirmar que la biología en la escuela entendida como una configuración que no supone la preexistencia de la ciencia biológica no ha estado siempre en la escuela ni ha permanecido inmutable.

Las prácticas discursivas relacionadas con la biología en la escuela colombiana se constituyen a propósito de formas de saber y fuerzas de poder que convergen en la escuela pero que también son producidas en ella. Asílo muestran los planes de estudio, los textos escolares, los informes de instrucción pública y las revistas, en los que se reconocen como líneas de ejercicio de poder (por los saberes que entroncan, las prácticas que incitan y los sujetos que constituyen) la alimentación, el niño, la ciencia, la educación, la enfermedad y el desarrollo. A todas ellas subyace la preocupación por la vida, donde el cuerpo y la población son objeto de producción e intervención, y la escuela es uno de los lugares privilegiados para ello.

Por tanto, se puede sugerir que la biología en la escuela no es el simulacro de la ciencia biológica, dado que sus prácticas son distintas y singulares. Este escrito, atendiendo al objetivo de esta convocatoria, visibiliza la emergencia, la permanencia y la transformación de la biología como saber escolar, como se muestra en los siguientes apartados.

\section{La genealogía de la biología en la escuela}

La célula animal es una pequeña porción de materia, blanda,

de dimensiones macroscópicas y de formas variables.

Sólo con la ayuda del microscópio han conseguido los naturalistas

observar este elemento primordial de todos los seres vivos.

\section{Bruño, Colección de Libros Escolares, 1912}

Se denomina "biología en la escuela" no con el ánimo de designar qué de la disciplina biológica se enseña, sino al contrario, visibilizar qué discursos y prácticas constituyen esa biología que se hace nominable en la escuela hacia principios de la década de 1930. Así, la mirada de este programa de investigación se enfoca en las condiciones que la hacen visible, es decir, en los discursos y prácticas que las constituyen, lo cual supone que la biología en la escuela no ha sido siempre la misma, pues tales prácticas discursivas son diferentes en el tiempo dadas sus formas de relación y las acciones de fuerza que les subyacen.

Pensar que la biología en la escuela no ha sido siempre la misma indica que la enseñanza supera la repetición y que se constituye en singularidad, en la que los sujetos, por ejemplo el maestro, no son solo 
reproductores, sino también generadores de formas, de acciones. En el presente trabajo es de gran relevancia este aspecto, pues no se pretende hacer juicios sobre qué tanta biología saben los maestros ni mucho menos señalar qué deben saber y para qué lo deben saber y si esto lo hace un profesional, sino mostrar qué sucede en ese acontecimiento de enseñanza, en el que se podría incluso sugerir que aquello que se denomina biología incluye las prácticas y discursos de la disciplina (los biólogos, el laboratorio o los tratados de biología), y además aquello que circula en otros lugares y que es producido por otros sujetos. Es, entonces, pertinente aclarar que al nombrar la escuela no se hace alusión al lugar - espacio físico-, sino a todas aquellas prácticas discursivas que a propósito de la educación se entrecruzan en y más allá de ella.

Es así que no se están buscando los conocimientos biológicos ni se pretende revisar su metodología de validación; la pregunta es por los saberes, por las formas que circulan y que se encuentran entroncadas en lo que se denomina biología. Saberes que pueden incluir dichos conocimientos pero no tienen mayor relevancia que aquellos que no son validados por la ciencia, pues el interés no es verificar, sino visibilizar todas las formas de saber que son condición de posibilidad de la biología en la escuela.

Se propone entonces volver atrás no sin extrañeza de aquello que parece inmutable. Es posible reconocer la singularidad de ciertas circunstancias que se configuran en posibilidades de recrear y potenciar. Es así que aquello que parece cierto y además repetitivo se hace único e irrepetible, es decir, nuevo, en palabras de Foucault: "actual". De este modo, la ciencia como forma de verdad y de conocimiento dado, como cierto per se, es acá cuestionada, es decir, se toma distancia de ella para visibilizar las fuerzas que la han posibilitado y las formas de saber que configura y que la han configurado. Por tanto, lo que se sugiere es problematizar las prácticas discursivas relacionadas con la biología y su enseñanza desde las relaciones entre saber, poder y subjetividad, con miras a hacer un análisis de lo que circula acerca de la biología en la escuela colombiana, el saber que se constituye sobre esta y la configuración de sujetos que posibilita.

Por tanto, se propone interrogar lo que aparece como realidad para el maestro de biología. Esto a través de una mirada histórica que lejos de establecer líneas de tiempo consiste en localizar la singularidad de los acontecimientos, en oponerse a las indefinidas teleologías y significados, así como a la búsqueda del origen, pues el objeto no es comprobar la existencia de lo ya dicho, las "solemnidades del origen" (Foucault, 2004, p. 19), es referirse a lo no dicho, por ello no se trata de constituir la verdad, ni el principio de la misma, sino de visibilizar la emergencia, los puntos de encuentro de fuerzas que constituyen acontecimientos.

Esta mirada es importante en la configuración del sujeto maestro, dado que el campo de la epistemología de la enseñanza de la biología, su historia y filosofía no se trabajan con mucha frecuencia en los programas de formación de pregrado de biología y licenciatura en biología, lo cual se evidencia en los programas actuales de formación de algunas universidades públicas y privadas.

Este cuestionamiento se relaciona con la noción del saber que abarca la pregunta por cómo se produce y cómo se legitima la verdad acerca de la enseñanza de la biología, es decir, por los modos de ser del conocimiento biológico y su relación con la pedagogía hoy. A este interrogante le subyacen las preguntas por la educación, la enseñanza, la escuela, el maestro y su subjetividad y el papel de la universidad.

\section{La emergencia de la biología en la escuela colombiana. La arqueología-genealogía: otra mirada a la biología en la escuela}

\author{
Las teorías que comento -y que veo por primera \\ vez expuestas en la obra \\ de este profesor madrileño-, buscan raíz en los \\ postulados mejor adquiridos \\ y más sólidos de la moderna biología. Inician un \\ plan que se apoya en esta
}

ciencia que día por día va explayando sus dominios, hasta terminar en el íntimo enlazamiento de

disciplinas que ayer parecían diversas y que hoy

se nos van

presentando conexas, y mañana nos dejarán columbrar la concreta unidad

de su origen y fines.

Robredo, 1921

La arqueología-genealogía se constituye en una mirada, dado que más que plantear un método propone una problematización sobre los modos de mirar. De esta manera, no señala una única forma de mirar, sino que deja al investigador la posibilidad de crear y recrear los objetos, es por ello que se asume como una caja de herramientas que tiene como uno de sus objetos el discurso, pero no pretende poner en evidencia su sentido, sino su funcionamiento como acontecimiento, $y$ establecer, del mismo modo, las relaciones que corresponden a diferentes distribuciones de los discursos y prácticas, así se sitúa en la dimensión de la historia, en el devenir de los acontecimientos y no de las estructuras 
Este escrito, entonces, señala una posible ruta de relaciones a propósito de la biología en la escuela, en la que "la biología en la escuela" es la visibilidad de prácticas discursivas que de ninguna manera son reductibles a dicha forma de nombrar, pero que hacen posible insinuar los enunciados que la conforman y dan emergencia a su visibilidad. Las condiciones indican el encuentro no determinado de formas de saber, fuerzas y sujetos que constituyen relaciones que posibilitan ciertas maneras de ver y formas de decir.

Así, la emergencia no se asume como principio u origen posible de fechar en tiempos y espacios únicos, sino como se muestra en los siguientes párrafos, estas relaciones provienen de distintos lugares que no hacen referencia a espacios físicos, sino a otros puntos de encuentro que se constituyen en fuerzas, en formas de saber que sin un único sentido o lógica convergen en la escuela.

Las condiciones de emergencia de la biología en la escuela colombiana se encuentran relacionadas con las prácticas discursivas a propósito de la enfermedad, la alimentación, la educación, la ciencia, el niño y el desarrollo. Estas son fuerzas de incitación y relación que configuran la visibilidad de la biología en la escuela, son solo una forma para expresar las relaciones alrededor de la preocupación por la vida, donde el organismo y su función son un objeto que establece vínculos entre evolución y raza, desarrollo y progreso.

El archivo muestra que la emergencia de la biología en la escuela colombiana está relacionada con la preocupación por la enfermedad, la alimentación y la mortalidad (señaladas por la estadística como problema), elementos que se suman a la noción de un clima tropical lleno de enfermedades y disminuido en oxígeno. De acuerdo con lo anterior, es a través de la educación que se pretende higienizar y hacer la profilaxis del cuerpo y el alma para posibilitar la vigorización racial y, por supuesto, el progreso y la civilidad a través de los cuales el ciudadano útil es el niño, la madre requiere ser educada para mantener la vida del hijo, el obrero y el pobre deben ser educados para disminuir el contagio y la mortalidad infantil.

Las prácticas y saberes de la biología en la escuela aluden a la alimentación, la enfermedad, la mortalidad, entre otros, a propósito de la vida. Pese a que estos temas ya eran preocupación desde finales del siglo XIX, como lo muestran las fuentes consultadas, la biología en la escuela se constituye en una configuración que distribuye de forma distinta los objetos y por tanto las relaciones con estos, en comparación con la historia natural y las ciencias de la naturaleza, ya que la biología reorganiza el objeto (vida -leyes naturales y sus reinos animal, vegetal, mineral- a propósito de lo vivo).
Asimismo, se involucran otras formas como los microorganismos (enfermedad-contagio), una noción que supera el cuerpo natural (reproduce, nutre y mueve) y lo traslada e integra (a través de la idea de organización, sensibilidad) a la noción de organismo que involucra la perspectiva evolutiva (que se relaciona con progreso, civilización, perfección, eugenesia, raza-especie, selección natural, desarrollo, adaptación), en la que la función desde la organización constituye el engranaje aparato-órganos, que involucra las leyes de la física y la química. De esta manera, la biología es nombrada como puente de relación con otras ciencias a propósito de su objeto de estudio - la vida- Así, lo vivo es organizado, se multiplica, se relaciona con el ambiente (preocupación por el agua y el aire) y cumple las funciones de respiración, nutrición y reproducción.

De este modo, la biología en la escuela emerge a propósito del objeto vida, ya que posibilita poner en relación el cuerpo con sus prácticas, la práctica del vivir. Prácticas discursivas en las que el organismo, como espacio de función y evolución, permite establecer desde la primera, la normalidad y el desarrollo y, desde la segunda, el progreso y la vigorización de la raza. De esta manera, la población se constituye en objeto de producción e intervención pues el organismo no es solo el individuo, sino la población es un organismo.

Por tanto, como ya se mencionó, la biología en la escuela reorganiza el objeto vida, es decir, este ya no es visto solo desde el cuerpo, pues además de incluir formas como las leyes naturales, el reino animal y el reino vegetal a través de la sospecha sobre el agua y el aire, involucra a los microorganismos por su relación enfermedad-contagio.

Así, la noción de cuerpo aparece inmersa dentro de las formas organismo y vida. De esta manera, la biología toma distancia de la historia natural distribuyendo de manera distinta lo que también eran saberes involucrados en esta, como la fisiología, la higiene, la química, entre otras. El archivo muestra el desplazamiento de la historia natural, que no indica su desaparición o una relación lineal con la biología; el desplazamiento está relacionado con el movimiento que sufre a propósito de prácticas discursivas que reconocen su necesidad en la escuela, lo que se hace visible en su poca mención en los discursos, en la paulatina disminución en la intensidad horaria en los planes de estudio.

La biología supera la noción del cuerpo natural caracterizado por reproducirse, nutrirse y moverse y lo traslada e integra a través de la idea de organización y sensibilidad a la noción de organismo. Esto involucra la perspectiva evolutiva que es relacionada con el 
progreso, la civilización, la perfección, la eugenesia, la raza, la especie, la selección natural, el desarrollo, y la adaptación.

La función desde la organización constituye el engranaje aparato-órganos, que puede explicarse desde las leyes de la física y la química, y la biología es puente de relación entre estas y otras ciencias (fisiología, anatomía, higiene) a propósito de su objeto de estudio, la vida - los seres vivos-. Ellos tienen una organización interna y externa que funciona y permite la reproducción, la nutrición y la respiración, en la que adquiere relevancia la relación con el ambiente (preocupación por el agua y el aire). Así, la biología en la escuela es una de las estrategias de biopoder que, según Foucault (1976), es “[...] un poder que ha tomado a su cargo el cuerpo y la vida, o que ha tomado a su cargo la vida en general, con un polo del lado del cuerpo y el otro polo del lado de la población". Según Albano (2005), en tal despliegue de fuerzas se produce una conjunción entre lo biológico y lo político, no solo por medio de prácticas sanitarias, sino por la instauración de complejas tecnologías de control biológico. De esta manera, dichas tecnologías posibilitan el control del cuerpo y la población, del hombre-cuerpo y el hombre-especie.

\section{Los intersticios: una genealogía de la biología en la escuela}

Las condiciones de emergencia como prácticas y discursos son, entonces, fuerzas de acción, formas de saber y sujetos que convergen, y producen formas de necesidad, verdad, justificación, acciones de incitación y limitación, al mismo tiempo que modos de ser sujetos, lo que da lugar a la biología en la escuela. La convergencia de estas prácticas se da con la vida como marco general.

Se procura la vida entendiendo desde dónde está es producida, de igual forma proponiéndola como fuerza de producción. Producción de formas, de prácticas, de juegos de relación, para el período en mención: enfermedad-alimentación, niño-perfección, desarrollo-raza, educación-progreso, ciencia-civilización, periodo en el que las prácticas están cruzadas y el procedimiento es ponerlas en relación, de tal modo que hay múltiples juegos de relación atravesados por las prácticas vinculadas con el cuerpo, la mortalidad, la higiene, la medicina, la inspección, que son también fuerzas de incitación, modos de producir, mantener y transformar los saberes y los sujetos; aparecen así la madre, el niño, el obrero, el pobre, el médico, el higienista, al lado de formas-fuerza como la naturaleza, la creación, el alma y el espíritu, que dan la pertinencia a espacios como la escuela, el hospital, la universidad, entre otros. Lugares que no son solo el espacio físico, sino que son configurados a través de las relaciones que los producen y que ellos producen.

De esta manera, son las prácticas discursivas relacionadas con la vida un modo de gobierno, una forma fuerza de relación, de conectar acciones y saberes, es fuerza de subjetivación. Entonces, la biología en la escuela como práctica y discurso que relaciona el cuerpo, la enfermedad y la alimentación, y tiene como medio el organismo, vinculado a la evolución y la herencia con el referente de la raza, provoca un modo de control tanto de individuos como de la población. Lo anterior debido a que involucra y representa la fuerza de incitación sobre la vida a través de formas, por ejemplo, el organismo que sugiere la conexión entre partes y la posibilidad de organización interna de estas, que se presenta como opción de organización externa, y da paso ahora a la población en cuanto organismo que puede controlar sus funciones, como la reproducción a través de regeneración de la raza, lo cual valida el discurso eugenésico; la función de respiración por medio del control de microbios a través del tratamiento del agua y el aire, a la vez que valida la sospecha sobre la población como agente contaminante que requiere la profilaxis y la higiene; la función de nutrición que señala que los niños están mal alimentados por la pobreza de sus familias, y el trabajo como obrero, lo que genera mortalidad por la no resistencia a enfermedades, es entonces cuando la biología en la escuela debe fomentar prácticas relacionadas con la buena alimentación y el aprovechamiento agrícola e industrial de las plantas.

Así, es visible la conexión que se hace entre estas prácticas discursivas y la biología en la escuela como modo de reagrupación de objetos, conceptos y acciones, una red que conecta y hace de ella, como práctica, una de las estrategias de control de la población, es decir, un modo de producir saber a la vez que se producen también acciones sobre la vida, lo cual podría sugerirse con referente hacia la actualidad para la sexualidad, la salud escolar, la educación ambiental, la biotecnología, entre otras prácticas, que son formas y acciones de la biología en la escuela hoy.

En la actualidad, aunque los enunciados son distintos, así como las relaciones saber, poder, subjetividad, se puede afirmar que el objeto de la biología en la escuela es la vida, no obstante, hablar de vida a comienzos del siglo pasado no es lo mismo que hablar de vida en los años 2000, así que se pretende continuar aportando en el campo intelectual de la biología en la escuela colombiana desde su historia y si es posible desde perspectivas comparadas.

Así, la historia de la biología en la escuela como programa de investigación posibilita tomar distancia 
de la inmediatez, de lo que aparece como realidad para el maestro de biología y hacer visible que la enseñanza como acontecimiento singular se configura en la confluencia de formas de saber, fuerzas de poder y modos de ser sujeto, es decir, prácticas discursivas, en las que el saber en cuanto categoría teórico-metodológica permite no reducir la mirada a los conocimientos biológicos, sino a todos los saberes que posibilitan la biología en la escuela, es decir, los conceptos involucrados, las instituciones que dan funcionamiento a estos y la subjetividad como singularidad y creación, pero también producción, donde las relaciones de poder no solo son de imposición, sino también de incitación.

De esta manera, la mirada genealógica a la biología en la escuela pone en cuestión la categoría conocimiento, como único objeto de investigación y señala otro elemento para el avance de la discusión del campo intelectual de la enseñanza de la biología - que es propósito de escuelas de formación de maestros en biología-, de este modo se visibiliza la importancia de la historia en la constitución del campo intelectual de la pedagogía y de la biología en la escuela, dado que como se ha señalado las investigaciones históricas no se realizan con el objeto de añorar el pasado o señalar el deber ser, sino que es un modo de problematizar las prácticas actuales, de visibilizar los modos en que se configuran prácticas discursivas y juegos de verdad sobre la biología y su enseñanza.

\section{Referencias bibliográficas}

Álvarez, A. (2003). La pedagogía y las ciencias: historia de una relación. En: Olga Lucía Zuluaga et al. Pedagogía y epistemología. Bogotá: Editorial Magisterio.

Álvarez, A. (2007). Ciencias sociales, escuela y nación: Colombia 1930-1960 (Tesis doctoral). Madrid: Universidad Nacional de Educación a Distancia.

Avanzini, G. (1990). La pedagogía desde el siglo XVII hasta nuestros días. México: Fondo de Cultura Económica.

Bauman, Z. (2007). Desafíos pedagógicos y modernidad líquida. Revista Propuesta Educativa, 16 (28), 7-18.

Benavot, A. (Mayo-agosto, 1991). El conocimiento para las masas: modelos mundiales y currícula nacionales. Revista de Educación, (295), 317-344.

Chevalard, Y. (1991). La transposición didáctica. Del saber sabio al saber enseñado. Buenos Aires: Aique.

Deleuze, G. (1987). Foucault. Barcelona: Paidós.

Diker, G. (2005). Los sentidos del cambio en educación. En G. Diker, y G. Frigerio, Educar: ese acto político. Buenos Aires: Del Estante.
Fiala, R. (2008). La ideología educativa y el currículo escolar. En A. Benavot y C. Braslavsky, El conocimiento escolar en perspectiva histórica y comparativa. Cambios de currículos en la educación primaria y secundaria (pp. 47-74). Buenos Aires: Granica.

Foucault, M. (1971). El polvo y la nube. En La imposible prisión: debate con Michel Foucault. Barcelona: Anagrama.

Foucault, M. (1981). La gubernamentalidad. En J. Varela y F. Álvarez-Uria, Espacios de poder (pp. 9-26). Paris: La Piqueta.

Foucault, M. (1991). Hacer vivir y dejar morir: la guerra como racismo. Madrid: Temps Modernes.

Foucault, M. (2002). La arqueología del saber. México: Siglo $\mathrm{xxI}$ editores.

Foucault, M. (2004). Nietzsche, la genealogía, la historia. Valencia: Pre-Textos.

Foucault, M. (2007). Las palabras y las cosas. Un arqueología de las ciencias humanas. México: Siglo XXI editores.

Goodson, I. (1994). Estudio del curriculum. Métodos y casos. Buenos Aires: Amorrortu.

Goodson, I. (2008). Procesos sociohistóricos de cambio curricular. En A. Benavot y C. Braslavsky, El conocimiento escolar en perspectiva histórica y comparativa. Cambios de currículos en la educación primaria y secundaria (pp. 335-350). Buenos Aires: Granica.

Herrera, C. (2007). De la escuela a la ciudad: Cuerpos civilizados, sujetos modernos. El caso colombiano en la primera mitad del siglo xx. En W. Moreno y S. Pulido, Educación, cuerpo y ciudad. El cuerpo en las interacciones e instituciones sociales (pp. 145-165). Medellín: Funámbulos.

Larrosa, J., y Jaramillo, A. (2007). La escuela: ¿Un modulador de subjetividad? En A. Marin, A. Agudelo y L. Escobar, Escuela, cuerpo y biopoder (pp. 8-26). Medellín: Alcaldía de Medellín.

Lazzarato, M. y Negri, A. (1997). Trabajo inmaterial y subjetividad. Recuperado de: http://www.nodo50.org/cdc/ Trabajoinmaterialysubjetividad.htm

Ledesma, I. (2008). Las prácticas médicas y la biología como ciencia: paradigmas, asimilación y domesticación social en México. Historia Ciencias Saude-Manguinho, 441-449.

Ledesma, I., y Barahona, A. (2004). La instucionalización de la biología en México. En: Asclepio, 56. Obtenido de http://historiamexicana.colmex.mx/pdf/13/ art_13_1873_16030.pdf

Lyotard, J. (1979). La condición posmoderna. Madrid: Cátedra.

Martínez, A.; Noguera, C. y Castro, J. (2003). Currículo y modernización. Cuatro décadas de educación en Colombia. Bogotá: Editorial Magisterio. 
Mayr, E. (2005). Así es la biología. Barcelona: Debate.

Mayr, E. (2006). Por qué es única la biología. Buenos Aires: Katz.

Noguera, C. (2003). Medicina y política. Medellín: Fondo Editorial Universitario.

Not, L. (2000). Las pedagogías del conocimiento. Bogotá: Fondo de Cultura Económica.

Popkewitz, T., Franklin, B., y Pereyra, M. (comp.) (2003). Historia cultural y educación: ensayos críticos sobre conocimiento y escolarización. Barcelona: Ediciones Pomares.

Roa, P. y Herrera, J. (2010). Historia de los saberes escolares: la emergencia de la biología en la escuela colombiana 1900-1930 (Tesis de maestría). Bogotá: Universidad Pedagógica Nacional.

Sáenz, J., Saldarriaga, O., y Ospina, A. (1997). Mirar la infancia: pedagogía, moral y modernidad en Colombia. Bogotá:
Colciencias, Foro por Colombia, Universidad de Antioquia, Uniandes.

Serres, M. (1991). Historia de las ciencias. Madrid: Editorial Cátedra.

Varela, J., y Álvarez-Uría, F. (1991). La arqueología de la escuela. Madrid: La Piqueta.

Viñao, A. (2006). La historia de las disciplinas escolares. Revista Interuniversitaria, (263).

Virno, P. (2003). Gramática de la multitud. Para un análisis de las formas de vida contemporáneas. Buenos Aires: Colihue S.R.L.

Zuluaga, O. (1999). Pedagogía e historia. La historicidad de la pedagogía. La enseñanza un objeto de saber. Bogotá: Anthropos.

Zuluaga, O. et al. (2003). Pedagogía y epistemología. Bogotá: Editorial Magisterio. 